\title{
A Validation Model for the Statistical Power Balance Method Using a Reverberation Chamber
}

\author{
Young Seung Lee ${ }^{1, *} \cdot$ Sung Woong Choi $^{2} \cdot$ Seung Keun Park ${ }^{1}$
}

\begin{abstract}
This paper proposes a validation model using a reverberation chamber for verification of the effectiveness and accuracy of the statistical power balance (PWB) method. The PWB method treats electromagnetic (EM) field distributions in certain environments as a representative value of an "ensemble" average, so a reverberation chamber is utilized as a testing apparatus to experimentally simulate the sets of measurement field "ensemble" inside a complex environment. Measurements are performed with various test conditions for environmental loss using flat absorbers, and front and side doors of the chamber. Comparison between the PWB analysis and the measurement results shows good agreement, indicating the usefulness of this statistical analysis method for stating the electromagnetic field inside the EM zone as a representative value to general structures.
\end{abstract}

Key Words: Ensemble Average, Mode Stirrer, Power Balance Method, Reverberation Chamber, Statistical Method.

\section{Introduction}

The trend toward increasing complexity of electronic devices and environments has made the computational burden of a realistic system for a full-wave electromagnetic (EM) analysis a growing concern. The power balance (PWB) method to assess statistical variations of EM fields was proposed as an efficient alternative method, in conjunction with the concept of the topology analysis [1], and its utility for large complex structures has recently been discussed [2] along with the theoretical background of statistical treatment. However, its validity over various amounts of power losses inside the same environment remains to be analyzed.

The aim of this paper is to propose an experimental validation model that uses a reverberation chamber to verify the availability of this statistical EM analysis method. Reverberation chambers have been widely used as test in the area of electromagnetic compatibility (EMC) measurements or for multi-input multi-output communications. We have focused the stochastic "ensemble" as the most fundamental feature of this chamber [3]. Measurements are performed under several non-ideal conditions, which correspond to the EM power losses in

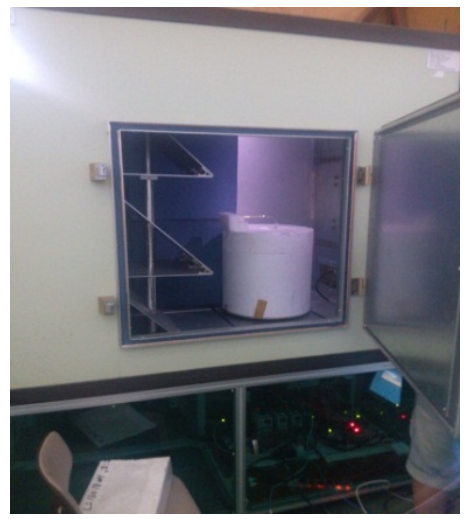

Fig. 1. A validation model for the power balance method using the reverberation chamber located at Korea Advanced Institute of Science and Technology.

each environment. The PWB method is validated by comparing the results with those of the PWB analysis of a proposed experimental model.

\section{A Validation Model and PWB Analysis}

The proposed model in this paper was developed using the reverberation chamber located at Korea Advan-

Manuscript received May 3, 2013 ; Revised June 25, 2013 ; Accepted July 18, 2013. (ID No. 20130503-025J)

${ }^{1}$ Radio Technology Research Department, Electronics and Telecommunications Research Institute, Daejeon, Korea.

${ }^{2}$ Broadcasting Systems Research Department, Electronics and Telecommunications Research Institute, Daejeon, Korea.

*Corresponding Author : Young Seung Lee (e-mail : lys009@etri.re.kr)

This is an Open-Access article distributed under the terms of the Creative Commons Attribution Non-Commercial License (http://creativecommons.org/licenses/ by-nc/3.0) which permits unrestricted non-commercial use, distribution, and reproduction in any medium, provided the original work is properly cited. 


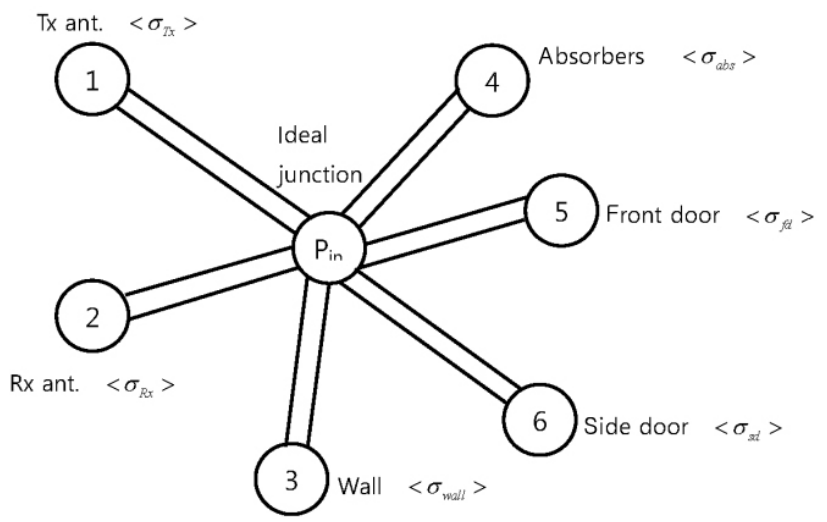

Fig. 2. Topology network representation of a proposed validation model.

ced Institute of Science and Technology. The chamber configuration is shown in Fig. 1. The chamber size is $1.8 \mathrm{~m} \times 1.2 \mathrm{~m} \times 1.4 \mathrm{~m}$. The vertical mode stirrer is present to the left of the main door. The flat absorber $(60$ $\mathrm{cm} \times 60 \mathrm{~cm})$, front door $(80 \mathrm{~cm} \times 80 \mathrm{~cm})$, and side door $(60 \mathrm{~cm} \times 60 \mathrm{~cm})$ are used to incorporate the EM losses in various situations. Detailed measurement conditions are discussed in Section III.

The PWB method outlined in [2] is used to characterize the proposed model shown in Fig. 1 by the topology network drawn in Fig. 2. The center ideal junction adds the total of the dissipated EM power, $P_{i n}$ is the incident feeding power from the transmitting antenna into the environment, and $\left\langle\sigma_{j}\right\rangle$ is the mean coupling cross section from each power loss mechanism $j$. The analytic expressions are well summarized in $[1,4]$, and are given as follows:

$$
\begin{aligned}
& <\sigma_{T x, R x}>=\frac{\lambda^{2}}{4 \pi} \alpha \eta_{T_{a}, R_{a}}\left(1-\left|S_{11}\right|^{2}\right) \\
& <\sigma_{\text {wall }}>=\frac{4 S}{3 c} \sqrt{\frac{\pi f \mu_{w}}{\mu_{0} \sigma_{w}}} \\
& <\sigma_{a b s}>=<\sigma_{f d, s d}>\approx \frac{A}{4}
\end{aligned}
$$

where $\lambda$ is the wavelength, $\eta_{T_{a}, R_{a}}$ is the radiation efficiency of the transmitting (Tx) and receiving $(\mathrm{Rx})$ antenna, $\alpha=1$ for $\mathrm{Tx}$ and $\alpha=2$ for $\mathrm{Rx}$ antenna, $S_{11}$ is its reflection coefficient, $S$ is total surface area of the chamber excluding apertures (doors and absorbers), $c$ is the speed of light, $f$ is the operating frequency, $\mu_{0}$ is the permeability of free space, $\mu_{w}$ is the permeability of the chamber wall, $\sigma_{w}$ is the conductivity of the chamber wall, and $A$ is the total cross-sectional area of apertures. Note that the corresponding adequate area should be used in $A$ of (3), according to the aperture loss me- chanism. The total mean coupling cross section $<\sigma_{t o t}>$ is obtained by the sum of all cross sections as:

$$
\begin{aligned}
& <\sigma_{t o t}>=<\sigma_{T x}>+<\sigma_{R x}>+<\sigma_{\text {wall }}> \\
& +<\sigma_{a b s}>+<\sigma_{f d}>+<\sigma_{s d}>
\end{aligned}
$$

and the mean power density $S_{c}$ inside the validation model can be computed from:

$$
S_{c}=\frac{P_{i n}}{\left.<\sigma_{t o t}\right\rangle} .
$$

\section{Measurement Results and Validations}

Measurement data were collected by rotating the mode stirrer through 2001 equally-spaced positions from a variety of test conditions. The experimental configuration is described in Fig. 3. Several aperture losses were added to the chamber model in order to increase cross section $\left\langle\sigma_{t o t}\right\rangle$ (equivalently, the total power loss) by making the use of the flat absorbers, and the front and side doors, and all measurement conditions are summa-

Table 1. All measurement conditions correspond to the total power loss

\begin{tabular}{cccc}
\hline Category & Wall A & Front door & Wall C \\
\hline Case 0 & - & - & - \\
Case 1 & 1 absorber & - & - \\
Case 2 & 2 absorbers & - & - \\
Case 3 & 4 absorbers & - & - \\
Case 4 & 4 absorbers & - & 2 absorbers \\
Case 5 & 4 absorbers & - & Side door opened \\
Case 6 & 4 absorbers & - & $\begin{array}{c}\text { Side door opened } \\
\text { with } 1 \text { absorber }\end{array}$ \\
Case 7 & 4 absorbers & Opened & - \\
Case 8 & 4 absorbers & Opened & 2 absorbers \\
Case 9 & - & - & 2 absorbers \\
\hline
\end{tabular}

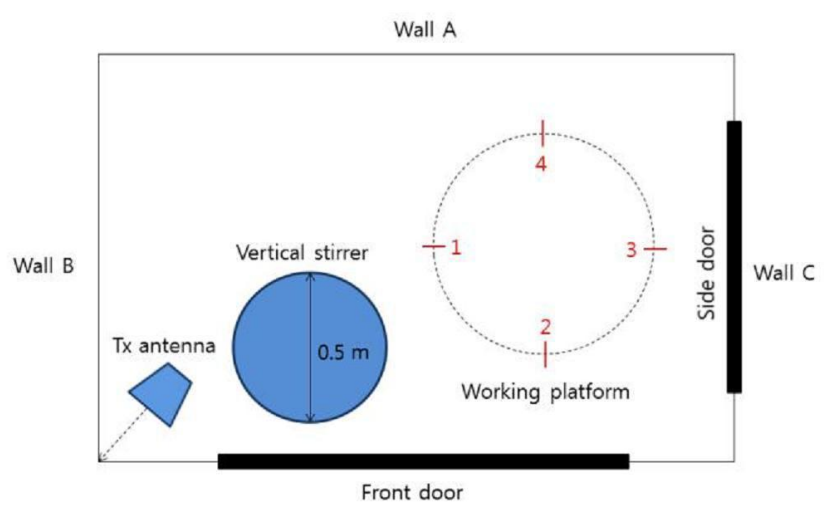

Fig. 3. Experimental configuration for a model. 
rized in Table 1. "Wall A" denotes the wall against the main front door and "Wall C" represents the wall to the right of the main door (against the vertical mode stirrer). The operating frequency in all experiments was set to $1.95 \mathrm{GHz}$. The Tx antenna was a double-ridged horn antenna and located at the left corner of the chamber. The Rx antenna aligned parallel to the vertical stirrer was a dipole antenna that has a resonance at $2 \mathrm{GHz}$ and located at four points in the working volume, along with a Styrofoam platform, as shown in Fig. 3. An Agilent PNA 8358A network analyzer was used to measure the insertion loss $S_{21}$ and the received power $P_{r}$. In the reverberation chamber, the mean power density $S_{c}$ is related to $P_{r}$ as

$$
\left\langle P_{r}\right\rangle=\frac{\lambda^{2}}{8 \pi} \eta_{R_{a}}\left(1-\left|S_{11}\right|^{2}\right) S_{c}
$$

where the symbol \langle\rangle denotes the ensemble average.

The measurement results for all conditions in Table 1 are plotted in Fig. 4. Also shown in the figure is the result of the PWB analysis from Eq. (5) for all experimental conditions. The large difference for the case 0 is mainly due to the insufficiency of the total power losses inside the environment for dissipating the feeding power [2]. Among the measurements, the curves are in good agreement except for the case of position 4, which shows a remarkable difference of a maximum $8 \mathrm{~dB}$ from the others. This is attributed to the fact that the measurement position is too close to the chamber wall and to the absorbers that incorporate the radiation losses; hence, the measurement is strongly influenced by the boundary field transition [5]. This correspondence allows the conclusion that the equivalence between the volume and ensemble average may be extended to the non-ideal lossy situation. The discrepancy between the PWB and the measurements also becomes more apparent as the environment losses increase, but it still shows relatively favorable correspondence within $10 \mathrm{~dB}$, which is an adequate safety margin in the area of EMC.

\section{Conclusion}

In this paper, we have proposed an experimental validation model that uses a reverberation chamber to verify the PWB method. The reverberation chamber can be used to simulate the statistical "ensemble" field and environment losses by employing a mode stirrer and changing the experimental conditions. Good agreement was found between the measurements and the PWB analysis, indicating the efficiency of this statistical method for the analysis of general complex structures.

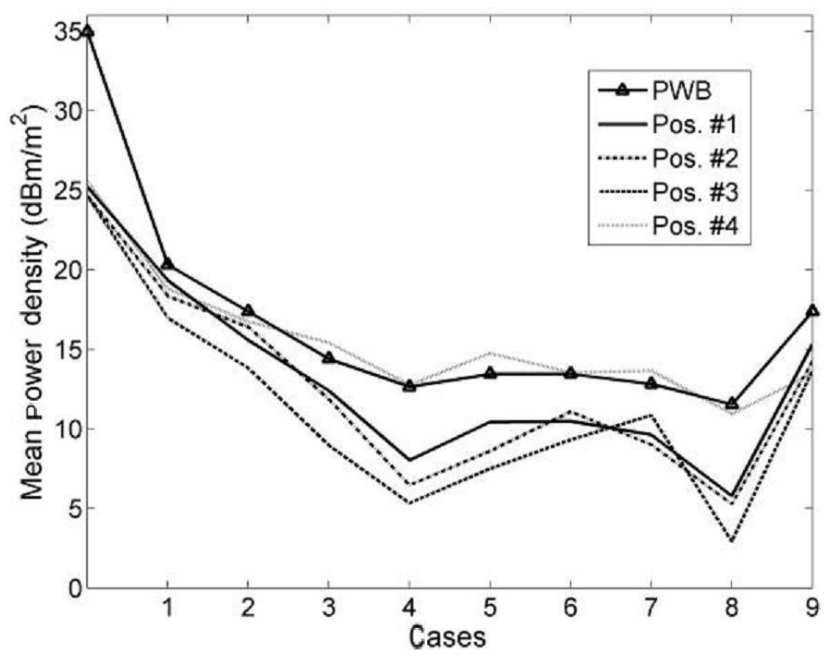

Fig. 4. Measurement and power balance (PWB) analysis results for all experimental conditions. Pos.: position.

This research was funded by the Ministry of Science, ICT \& Future Planning, Korea in the ICT R\&D Program 2013. The author wishes to express his deep gratitude to Mr. D. Sim for his helpful advice on a number of results regarding validation of a reverberation chamber during the study of this paper. The special assistance of Mr. M. Jung and Mr. B. Park is also gratefully appreciated.

\section{References}

[1] I. Junqua, J. P. Parmantier, and F. Issac, "A network formulation of the power balance method for highfrequency coupling," Electromagnetics, vol. 25, no. 7-8, pp. 603-622, Feb. 2007.

[2] Y. S. Lee and S. K. Park, "A study on the utility of statistical power balance method for efficient electromagnetic analysis of large and complex structures," Journal of the Korea Electromagnetic Engineering Society, vol. 24, no. 2, pp. 189-197, Feb. 2013.

[3] Electromagnetic Compatibility (EMC) Part 4-21: Testing and Measurement Techniques - Reverberation ChamBer Test Methods, IEC 61000-4-21, 2001.

[4] I. Junqua and J. P. Parmantier, "Power balance modeling techniques," Presented at Young Scientists' Programme at EMC EUROPE 2010, Wroclaw, Poland, Sep. 2010.

[5] D. A. Hill, Electromagnetic Fields in Cavities: Deterministic and Statistical Theories, Piscataway, NJ: IEEE, 2009. 